\title{
Clinical course and disease outcome in patients with severe COVID-19 receiving remdesivir
}

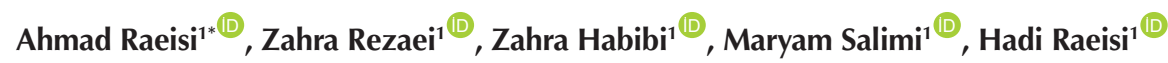 \\ ${ }^{1}$ Clinical Research Development Unit, Hajar Hospital, Shahrekord University of Medical Sciences, Shahrekord, Iran
}

*Corresponding Author: Ahmad Raeisi, Hajar Hospital, Shahrekord University of Medical Sciences, Shahrekord, Iran. Tel: +9809232841594, Email: draraeisi88@yahoo.com

\begin{abstract}
Background and aims: COVID-19 is a pandemic and infectious disease with high morbidity and mortality rates as well as a global spread. This study aimed to investigate the clinical outcomes for patients afflicted with COVID-19 and treated with remdesivir.

Methods: In this clinical trial study, patients with severe COVID-19 confirmed by molecular testing and hospitalized at Hajar Hospital of Shahrekord in Iran were divided into standard care group (including 52 patients) and standard + remdesivir group (including 51 patients). The patients were examined for clinical symptoms, laboratory data, as well as mortality and recovery rates during the treatment period. Then the data were analyzed by SPSS version 23 using chi-square, independent t-test and repeated-measures ANOVA.

Result: Clinical and laboratory monitoring on days 5, 10, and 14 showed that there was no significant difference between two groups $(P>0.05)$. In addition, there was no significant difference between the two groups in terms of hospitalization duration and ICU rate (58.8\% versus $51.9 \%, P=0.48)$. The trend of Lymph count and platelet decreased significantly in the standard group $(P=0.003, P=0.04$, and $P=0.03$, respectively).

Conclusion: Patients with severe COVID-19 were investigated and it was concluded that remdesivir produced no improvement in the remdesivir group compared to the standard group.

Keywords: COVID-19, Treatment, Hospitalization
\end{abstract}

Received: 27 April 2021, Accepted: 9 June 2021, ePublished: 26 January 2022

\section{Introduction}

COVID-19 is a pandemic and infectious disease that is caused by a virus from the Coronaviridae family. Coronavirus is a single-stranded, RNA-carrying virus and the seventh member of the given family, which often affects animals - including birds and mammals - and causes mild respiratory infections like a common cold in humans; however, human coronaviruses can lead to deadly endemics (1). COVID-19 infection symptoms range from asymptomatic to septic shock. The most common clinical findings are fever, fatigue, dry cough, sore throat, and diarrhea. The infection is classified into mild, moderate, severe, and vital. The mild group includes patients with mild upper respiratory tract infections symptoms like dry cough, mild fever, sore throat, headache, and myalgia without symptoms like dyspnea and radiological manifestations. The moderate group generally presents as respiratory symptoms like cough, dyspnea, and tachycardia with radiological manifestations $(2,3)$. The severe group exhibits at least one of the three symptoms: 1 . dyspnea and respiratory rate $>30$ breaths per minute ( $R R>30$ times/min); 2. arterial blood oxygen to fraction of inspired oxygen ratio less than 300 ( $\mathrm{Pao} 2$ / Fio2 <300); and 3. blood oxygen saturation levels less than $90 \%$ (Spo $2<93 \%$ ). Finally, the vital condition group manifests itself in at least one of the three symptoms: respiratory failure, septic shock requiring mechanical ventilation, or along with other organ failure, requiring admission to intensive care unit Multiple organ failure (4). Misra et al have shown that the mortality risk of infection varies in different people. The risk is higher in people with underlying diseases. Hypertension, cardiovascular disease, diabetes mellitus, and respiratory diseases are the most important comorbidities of COVID-19 (5). Madsen et al have reported that remdesivir can shorten the recovery time in patients with COVID-19, and presented evidence for lower respiratory tract infection (6).

Remdesivir is a monophosphate prodrug whose active metabolism is an analog of adenosine triphosphate. The active drug inhibits RNA-dependent RNA polymerase (RdPp) in Coronaviridae and Flaviviridae families. Infusion of up to $225 \mathrm{mg}$ of the drug is tolerated well. It does not require renal or hepatic dose adjustment, but is not recommended in patients with a glomerular filtration rate lower than $30 \mathrm{~mL} / \mathrm{min}$ (glomerular filtration rate $<30$ $\mathrm{mL} / \mathrm{min}$ ) (7). Despite the numerous studies concerning the effect of this drug on COVID-19 disease, as well as due to the differences found in people regarding genetic/ epigenetic properties and in health care systems, this study aimed to examine the remdesivir in hospitalized COVID-19 patients in Shahrekord, Chaharmahal Bakhtiari province, south west Iran. 


\section{Materials and Methods}

\section{Patients}

Inclusion criteria for the present study were: hospitalized patients aged 18 years or over with the SARS-CoV-2 infection confirmed by molecular test; those having blood oxygen saturation $\leq 93 \%$ in room air or needing supportive oxygen/ventilation; those with pulmonary infiltration detected by radiological studies.

Patients with alanine aminotransferase and aspartate aminotransferase rising five times higher than the upper limit of normal rate and those with Glomerular filtration rate less than $30 \mathrm{~mL} / \mathrm{min}$ (according to Cockroft-Gault formula) based on hospital protocol were excluded from the study.

\section{Study design}

In this clinical trial study, 100 patients hospitalized in Hajar Hospital, Shahrekord, Iran were divided into standard and remdesivir groups in a ratio of 1:1. The study sampling was nonequivalence and non-randomized.

Due to the limited access to remdesivir and in order to comply with ethical principles, patients hospitalized in drug availability courses were eligible to receive remdesivir, but patients admitted in other courses only received standard treatments based on the national protocol (8). Patients in the remdesivir group received remdesivir 200 mg intravenous (BDREM-100 Company, India) on the first day and, then, received $100 \mathrm{mg}$ intravenous infusion in 15-30 minutes for the following four days. Remdesivir prescription was stopped for patient meeting the exclusion criteria. All patients were assessed on the $1^{\text {st }}, 5^{\text {th }}, 10^{\text {th }}$, and $14^{\text {th }}$ days. In addition, patients' examination period lasted for three months.

\section{Clinical and laboratory monitoring}

Patients' assessment included clinical sign blood oxygen saturation, oxygen supplementary device, body temperature, cough, dyspnea, gastrointestinal symptoms (e.g., nausea and vomiting, diarrhea, abdominal pain), headache, and myalgia. On days 1, 5, 10, and 14, blood samples were collected for measuring the blood cell count, liver enzymes (aspartate transaminase [AST], alanine transaminase [ALT], alkaline phosphatase [ALP]), total bilirubin, prothrombin time, partial thromboplastin time, international normalized ratio (INR), and inflammatory factors (lactate dehydrogenase, erythrocyte sedimentation ratio) and creatinine.

\section{Outcome}

The first endpoint of the study was the discharge of hospital or death of patients, and the secondary endpoint was adverse effect for patients throughout the duration of study. Patients hospitalized up to day 14 or those remained hospitalized after this period until final status determination (i.e., death or discharge) were all under observation. The study's important outcomes included hospitalization duration, ICU admission, mortality, the interval between onset of symptom, and the start of therapy with remdesivir and recovery. Recovery was defined as getting discharged in two weeks (14 days) or hospitalization without oxygen supplementary on day 14 .

\section{Statistical analysis}

The data were analyzed by using SPSS software (version 23) and performing chi square test, independent t-test and repeated-measures ANOVA. In all tests, a $P$ value less than 0.05 was considered statistically significant.

\section{Results}

Out of 103 patients meeting inclusion criteria, 52 ones received only standard treatment and 51 ones received remdesivir. Patients hospitalized during the period when remdesivir was available received this drug in addition to standard treatment, while other patients only received the standard care. Patients' demographic information and the information on their underlying disease were recorded at the beginning of the study.

The mean age for remdesivir group was $62.2 \pm 16.7$ while it was $51.7 \pm 11.7$ for control group, which was significantly different $(P<0.001)$. The proportion of females was $55.8 \%$ versus males $44.2 \%(P=0.02)$.

Hypertension (HTN), diabetes, and cardiovascular disease were the most common underlying diseases found in both groups; however, no difference was detected between the two groups in this regard $(P>0.05)$. The number of chronic respiratory and neurological diseases observed in the standard group was significantly higher than that in the remdesivir group (Table 1).

Clinical symptoms like fever, cough, dyspnea, gastrointestinal symptoms, and other symptoms were monitored during hospitalization. Clinical and laboratory monitoring on days 5,10 , and 14 revealed that there was no significant difference between the two groups regarding the given symptoms (Tables 2 and 3 ).

The highest rate of fever was recorded on the $1^{\text {st }}$ day and the least one was observed on the $10^{\text {th }}$ day $(0 \%)$ of the study in both groups. The highest prevalence of cough and dyspnea in both groups was recorded on the $1^{\text {st }}$ day while the least one was observed on the $10^{\text {th }}$ and $14^{\text {th }}$ days in standard and remdesivir groups, respectively.

Gastrointestinal symptoms and other symptoms prevalence were most common on the $1^{\text {st }}$ and $4^{\text {th }}$ days in the standard and remdesivir groups, whereas the lowest prevalence of the given symptoms was observed on the $14^{\text {th }}$ and $10^{\text {th }}$ days in the standard and remdesivir groups, respectively.

White blood cell count increased until day 14 in the remdesivir group but it increased until day 10 in the standard group, compared to its minimum count on the $1^{\text {st }}$ day; however, no significant difference was found between the two groups in this regard.

Platelet counts in the standard group significantly changed during the hospitalization period and the highest platelet count in this group was observed on the $10^{\text {th }}$ day. 
Table 1. Comparison of demographic/disease variables in standard and remdesivir groups

\begin{tabular}{|c|c|c|c|c|}
\hline Variable & Subgroup & Standard & Remdesivir & $P$ value $^{a}$ \\
\hline \multirow{2}{*}{ Gender } & Male & $23(44.20)$ & $34(66.7)$ & \multirow{2}{*}{$0.02 *$} \\
\hline & Female & $29(55.8)$ & $23(44.2)$ & \\
\hline \multirow{2}{*}{$\begin{array}{l}\text { Cardiovascular } \\
\text { disease }\end{array}$} & Positive & $8(15.40)$ & $6(11.80)$ & \multirow{2}{*}{0.59} \\
\hline & Negative & $44(84.60)$ & $45(88.20)$ & \\
\hline \multirow{2}{*}{$\begin{array}{l}\text { Chronic respiratory } \\
\text { disease }\end{array}$} & Positive & $9(17.30)$ & $2(3.90)$ & \multirow{2}{*}{$0.03 *$} \\
\hline & Negative & $43(82.70)$ & $29(96.10)$ & \\
\hline \multirow{2}{*}{ Chronic renal disease } & Positive & $2(3.80)$ & $0(0)$ & \multirow{2}{*}{0.50} \\
\hline & Negative & $50(96.20)$ & $51(100)$ & \\
\hline \multirow{2}{*}{ Diabetes mellitus } & Positive & $14(27.50)$ & $11(21.60)$ & \multirow{2}{*}{0.49} \\
\hline & Negative & $37(72.50)$ & $40(78.40)$ & \\
\hline \multirow{2}{*}{ Hypertension } & Positive & $22(42.30)$ & $15(29.40)$ & \multirow{2}{*}{0.17} \\
\hline & Negative & $30(57.70)$ & $36(70.60)$ & \\
\hline \multirow{2}{*}{ Liver disease chronic } & Positive & $1(1.90)$ & $0(0)$ & \multirow{2}{*}{0.99} \\
\hline & Negative & $51(98.10)$ & $51(100)$ & \\
\hline \multirow{2}{*}{ Immunocompromised } & Positive & $1(1.90)$ & $0(0)$ & \multirow{2}{*}{0.99} \\
\hline & Negative & $51(98.10)$ & $51(100)$ & \\
\hline \multirow{2}{*}{ Neural disease } & Positive & $8(15.70)$ & $2(3.90)$ & \multirow{2}{*}{$0.04 *$} \\
\hline & Negative & $43(84.30)$ & $49(96.10)$ & \\
\hline \multirow{2}{*}{ Other diseases } & Positive & $18(34.60)$ & $10(19.60)$ & \multirow{2}{*}{0.09} \\
\hline & Negative & $34(65.40)$ & $41(80.40)$ & \\
\hline
\end{tabular}

${ }^{a}$ Chi-square test; $*$ Significant at $P<0.05$.

The changes were not significant in the remdesivir group.

Measurement analysis showed that the trend of Lymph and Platelet counts decreased significantly in the standard group ( $P=0.003, P=0.04$, and $P=0.03$, respectively).

Patients' respiratory status in terms of using the respiratory devices during the studies period is present in Figure 1.

As for the standard group, the most commonly-used device was a reserve bag on days 1,5 , and 10 , but the patients' status changed into the discharged status on day 14. As for the remdesivir group, most patients used reserve bags on days 1 and 5, but the discharged status was the most common status observed on days 10 and 14 .

Patients receiving remdesivir were divided into two groups $\leq 10$ and $>10$ based on the interval between the onset of symptoms and the start of treatment with remdesivir. This factor caused no difference in mortality rate as well as in gastrointestinal or other symptoms in the recipients of the remdesivir (Figure 2).

No significant difference was detected between the standard group and remdesivir one regarding the rate of mortality and discharge for patients with HTN, diabetes, and cardiovascular disease as the most common underlying diseases (Figure 3).

Hospitalization duration were $12.8 \pm 8.5$ and $12.75 \pm 7.5$ for the remdesivir group and control group, respectively, which were not significant $(P=0.98)$.

ICU admission rate for the two groups was not statistically different (Table 4).

Recovery was defined in terms of discharge or respiration without the need for respiratory assistance device on day 14 , whereby the difference was not significant.(80.4 versus 69.2; $P=0.19$ ).

\section{Discussion}

Remdesivir had no significant effect on mortality rate, hospitalization duration, and ICU admission; and they were not even affected by sex, comorbidities, and interval

Table 2. Comparison of clinical symptoms in standard and remdesivir groups

\begin{tabular}{|c|c|c|c|c|}
\hline Variable & Subgroup & Standard & Remdesivir & $P$ value \\
\hline \multirow{2}{*}{ Fever day 1} & Positive & $17(32.70)$ & $6(12.50)$ & \multirow{2}{*}{$0.02^{*}$} \\
\hline & Negative & $35(67.30)$ & $42(87.50)$ & \\
\hline \multirow{2}{*}{ Fever day 5} & Positive & $1(2.00)$ & $2(4.80)$ & \multirow{2}{*}{0.59} \\
\hline & Negative & $48(98.00)$ & $40(95.20)$ & \\
\hline \multirow{2}{*}{ Fever day 10} & Positive & $0(0)$ & $0(0)$ & \multirow{2}{*}{ - } \\
\hline & Negative & $29(100)$ & $21(100)$ & \\
\hline \multirow{2}{*}{ Fever day 14} & Positive & $1(5.30)$ & $0(0)$ & \multirow{2}{*}{0.99} \\
\hline & Negative & $18(94.70)$ & $13(100)$ & \\
\hline \multirow{2}{*}{ Cough day 1} & Positive & $32(61.50)$ & $34(68.00)$ & \multirow{2}{*}{0.54} \\
\hline & Negative & $20(38.50)$ & $16(32.00)$ & \\
\hline \multirow{2}{*}{ Cough day 5} & Positive & $22(50.00)$ & $14(36.80)$ & \multirow{2}{*}{0.23} \\
\hline & Negative & $22(50.00)$ & $24(63.20)$ & \\
\hline \multirow{2}{*}{ Cough day 10} & Positive & $6(23.10)$ & $7(41.20)$ & \multirow{2}{*}{0.21} \\
\hline & Negative & $20(76.90)$ & $10(58.80)$ & \\
\hline \multirow{2}{*}{ Cough day 14} & Positive & $9(40.00)$ & $9(25.00)$ & \multirow{2}{*}{0.68} \\
\hline & Negative & $15(60.00)$ & $12(75.00)$ & \\
\hline \multirow{2}{*}{ Dyspnea day 1} & Positive & $39(75.00)$ & $32(64.00)$ & \multirow{2}{*}{0.23} \\
\hline & Negative & $13(25.00)$ & $18(36.00)$ & \\
\hline \multirow{2}{*}{ Dyspnea day 5} & Positive & $18(40.90)$ & $10(26.30)$ & \multirow{2}{*}{0.17} \\
\hline & Negative & $26(59.10)$ & $28(73.70)$ & \\
\hline \multirow{2}{*}{ Dyspnea day 10} & Positive & $5(19.20)$ & $6(35.30)$ & \multirow{2}{*}{0.30} \\
\hline & Negative & $21(80.80)$ & 17 (64.70) & \\
\hline \multirow{2}{*}{ Dyspnea day 14} & Positive & $4(28.60)$ & $2(16.70)$ & \multirow{2}{*}{0.65} \\
\hline & Negative & $10(71.40)$ & $10(83.30)$ & \\
\hline \multirow{2}{*}{$\begin{array}{l}\text { Gastrointestinal sign } \\
\text { day } 1\end{array}$} & Positive & $21(40.40)$ & $14(28.00)$ & \multirow{2}{*}{0.19} \\
\hline & Negative & $31(59.60)$ & $36(72.00)$ & \\
\hline Gastrointestinal sign & Positive & $6(13.30)$ & $2(5.30)$ & 028 \\
\hline day 5 & Negative & $39(86.70)$ & $36(94.70)$ & 0.20 \\
\hline Gastrointestinal sign & Positive & $2(7.70)$ & $0(0)$ & 01 \\
\hline day 10 & Negative & $24(92.30)$ & $17(100)$ & 0.01 \\
\hline Gastrointestinal sign & Positive & $0(0)$ & $1(8.30)$ & $0: 48$ \\
\hline day 15 & Negative & $13(100)$ & $11(91.70)$ & \\
\hline Othe & Positive & $31(59.60)$ & $17(34.00)$ & 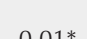 \\
\hline 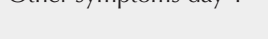 & Negative & $21(40.40)$ & $33(66.00)$ & \\
\hline & Positive & $5(13.30)$ & $3(7.90)$ & \\
\hline 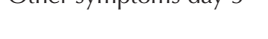 & Negative & $39(86.70)$ & $35(92.10)$ & \\
\hline & Positive & $1(3.80)$ & $0(0.0)$ & \\
\hline Give sympromis uay 10 & Negative & $25(96.20)$ & $17(100)$ & 0.97 \\
\hline & Positive & $0(0)$ & $1(8.30)$ & \\
\hline & Negative & 13(100) & $11(91.70)$ & \\
\hline
\end{tabular}

${ }^{a}$ Chi-square test; $*$ Significant at $P<0.05$ 
Table 3. Comparison of the average scores of of laboratory data in standard and remdesivir groups

\begin{tabular}{|c|c|c|c|c|c|}
\hline \multirow{2}{*}{ Variable } & \multicolumn{2}{|c|}{ Remdesivir } & \multicolumn{2}{|c|}{ Standard } & \multirow{2}{*}{$P$ value $^{\mathrm{a}}$} \\
\hline & SD & Mean & SD & Mean & \\
\hline WBC day 1 & 8787.73 & 3799.37 & 330.59 & 7193.88 & $0.04^{*}$ \\
\hline WBC day 5 & 12002.23 & 11041.03 & 2903.30 & 8128.57 & 0.48 \\
\hline WBC day 10 & 4492.49 & 11104.76 & 353.31 & 11065.00 & 0.72 \\
\hline WBC day 14 & 20009.89 & 16069.23 & 3397.98 & 9268.75 & 0.45 \\
\hline$P$ value intra group & \multicolumn{2}{|c|}{0.21} & \multicolumn{2}{|c|}{0.04} & \\
\hline Lymph count day 1 & 614.12 & 935.78 & 1441.96 & 1345.11 & 0.08 \\
\hline Lymph count day 5 & 896.30 & 1024.20 & 884.29 & 928.26 & 0.65 \\
\hline Lymph count day 10 & 332.09 & 836.49 & 564.29 & 764.92 & 0.62 \\
\hline Lymph count day 14 & 2321.59 & 1461.90 & 362.34 & 449.86 & 0.10 \\
\hline$P$-value intra group & \multicolumn{2}{|c|}{0.25} & \multicolumn{2}{|c|}{0.04} & \\
\hline Platelet day 1 & 80.36 & 207.34 & 81.27 & 189.76 & 0.30 \\
\hline Platelet day 5 & 116.44 & 249.15 & 99.99 & 216.06 & 0.97 \\
\hline Platelet day 10 & 96.00 & 198.95 & 104.46 & 226.95 & 0.13 \\
\hline Platelet day 14 & 73.87 & 161.38 & 66.01 & 146.81 & 0.98 \\
\hline$P$ value intra group & \multicolumn{2}{|c|}{0.23} & \multicolumn{2}{|c|}{0.03} & \\
\hline AST day 1 & 32.78 & 52.20 & 27.64 & 46.60 & 0.38 \\
\hline AST day 5 & 21.90 & 46.30 & 25.87 & 46.56 & 0.77 \\
\hline AST day 10 & 50.28 & 45.73 & 21.60 & 47.25 & 0.99 \\
\hline AST day 14 & 89.58 & 63.36 & 570.51 & 243.07 & 0.21 \\
\hline$P$ value intra group & \multicolumn{2}{|c|}{0.60} & \multicolumn{2}{|c|}{0.19} & \\
\hline ALT day 1 & 34.35 & 50.38 & 24.07 & 36.98 & $0.04^{*}$ \\
\hline ALT day 5 & 54.23 & 76.98 & 185.70 & 80.65 & 0.08 \\
\hline ALT day 10 & 165.42 & 101.00 & 40.34 & 65.45 & 0.37 \\
\hline ALT day 14 & 55.28 & 63.91 & 431.42 & 196.73 & 0.24 \\
\hline$P$ value intra group & \multicolumn{2}{|c|}{0.86} & \multicolumn{2}{|c|}{0.19} & \\
\hline ALP day 1 & 116.10 & 127.38 & 49.18 & 115.00 & 0.56 \\
\hline ALP day 5 & 136.16 & 14.93 & 44.61 & 116.96 & 0.78 \\
\hline ALP day 10 & 72.14 & 128.09 & 47.30 & 126.68 & 0.47 \\
\hline ALP day 14 & 53.98 & 115.70 & 32.94 & 112.85 & 0.43 \\
\hline$P$-value intra group & \multicolumn{2}{|c|}{0.59} & \multicolumn{2}{|c|}{0.82} & \\
\hline PT day 1 & 9.61 & 14.30 & 2.36 & 13.17 & 0.45 \\
\hline PT day 5 & 7.85 & 15.53 & 8.78 & 17.58 & 0.55 \\
\hline PT day 10 & 6.36 & 15.89 & 4.69 & 15.55 & 0.81 \\
\hline PT day 14 & 10.36 & 19.55 & 9.19 & 19.11 & 0.26 \\
\hline$P$ value intra group & \multicolumn{2}{|c|}{0.20} & \multicolumn{2}{|c|}{0.29} & \\
\hline PTT day 1 & 3.26 & 29.56 & 13.86 & 30.92 & 0.63 \\
\hline PTT day 5 & 22.65 & 39.91 & 4.55 & 31.24 & 0.15 \\
\hline PTT day 10 & 19.91 & 38.71 & 2.26 & 29.25 & 0.08 \\
\hline PTT day 14 & 9.43 & 38.77 & 12.55 & 35.93 & 0.44 \\
\hline$P$ value intra group & \multicolumn{2}{|c|}{0.11} & \multicolumn{2}{|c|}{0.69} & \\
\hline INR day 1 & 0.11 & 1.05 & 0.24 & 1.12 & 0.17 \\
\hline INR day 5 & 0.97 & 1.44 & 0.940 & 1.46 & 0.28 \\
\hline INR day 10 & 1.34 & 1.65 & 0.54 & 1.37 & 0.25 \\
\hline INR day 14 & 0.98 & 1.49 & 0.73 & 1.49 & 0.28 \\
\hline$P$ value intra group & \multicolumn{2}{|c|}{0.39} & \multicolumn{2}{|c|}{0.07} & \\
\hline ESR day 1 & 30.74 & 39.90 & 25.95 & 35.05 & 0.60 \\
\hline ESR day 5 & 18.38 & 61.00 & 10.65 & 21.25 & 0.87 \\
\hline
\end{tabular}


Table 3. Continued

\begin{tabular}{|c|c|c|c|c|c|}
\hline \multirow{2}{*}{ Variable } & \multicolumn{2}{|c|}{ Remdesivir } & \multicolumn{2}{|c|}{ Standard } & \multirow{2}{*}{$P$ value $^{\mathrm{a}}$} \\
\hline & SD & Mean & SD & Mean & \\
\hline ESR day 10 & 00.00 & 57.00 & 8.73 & 13.67 & --- \\
\hline ESR day 14 & 10.60 & 16.50 & --- & --- & --- \\
\hline$P$ value intra group & \multicolumn{2}{|c|}{--} & \multicolumn{2}{|c|}{0.60} & \\
\hline LDH day 1 & 301.11 & 622.85 & 203.53 & 448.64 & $0.006^{*}$ \\
\hline LDH day 5 & 538.47 & 682.59 & 414.49 & 620.53 & 0.87 \\
\hline LDH day 10 & 401.64 & 787.69 & 342.38 & 597.85 & 0.77 \\
\hline LDH day 14 & 401.95 & 557.13 & 253.86 & 537.63 & 0.87 \\
\hline$P$ value intra group & \multicolumn{2}{|c|}{0.32} & \multicolumn{2}{|c|}{0.28} & \\
\hline Creatinine day 1 & 0.20 & 0.81 & 0.30 & 0.91 & 0.06 \\
\hline Creatinine day 5 & 0.23 & 0.78 & 0.24 & 0.79 & 0.16 \\
\hline Creatinine day 10 & 0.53 & 0.89 & 0.32 & 0.80 & 0.07 \\
\hline Creatinine day 14 & 4.87 & 2.27 & 0.53 & 0.81 & 0.42 \\
\hline$P$ value intra group & \multicolumn{2}{|c|}{0.26} & \multicolumn{2}{|c|}{0.36} & \\
\hline Direct bilirubin day 1 & 0.21 & 0.26 & 0.12 & 0.21 & 0.14 \\
\hline Direct bilirubin day 5 & 0.29 & 0.27 & 0.17 & 0.25 & 0.66 \\
\hline Direct bilirubin day 10 & 0.24 & 0.30 & 1.02 & 0.25 & 0.42 \\
\hline Direct bilirubin day 14 & 1.04 & 0.68 & 0.15 & 0.30 & 0.99 \\
\hline$P$ value intra group & \multicolumn{2}{|c|}{0.91} & \multicolumn{2}{|c|}{0.14} & \\
\hline
\end{tabular}

a Intergroup Independent t-test results; $*$ Significant at $P<0.05$.

Table 4. Comparison of hospitalization and ICU admission in standard and remdesivir groups

\begin{tabular}{lcccc}
\hline Variable & Remdesivir & Standard & P value (Chi-square test) \\
\hline Hospitalized duration & $12.8 \pm 8.5$ & $12.82 \pm 7.5$ & 0.98 \\
ICU & No & $30(58.8)$ & $27(51.9)$ & 0.48 \\
& Yes & $21(41.2)$ & $25(48.1)$ & \\
\hline
\end{tabular}

between symptom onset and remdesivir prescription. In a study by Wang et al in Wuhan, it was determined that remdesivir had no impact on mortality rate and clinical recovery time (3); while in the study by Olender et al, it was shown that remdesivir significantly reduced the mortality rate compared to the standard group. Also, Tomashek et al found that hospitalization duration and mortality rate in the remdesivir group on days 5 and 29 were less than those observed in the standard group $(6,9)$.

Goldman and colleagues' study found no significant difference among remdesivir treated groups in terms of mortality rate and hospitalization duration between days 5 to 10 , although the discharge rate was higher in remdesivir treated group on the $5^{\text {th }}$ day (10). Taking into account Goldman and colleagues' results concerning the insignificant difference in mortality and hospitalization period after using remdesivir for 5 days, it seemed that using remdesivir for 5 days in our study could not have also been an important factor in remdesivir efficacy.

In another study by Grein et al, no difference was observed among patients receiving remdesivir with invasive or non-invasive oxygen supplementary (11). In this study, the most common underlying diseases among patients in both groups were HTN and diabetes mellitus. The most common comorbidities were the same ones found in Wang's study, while cardiovascular diseases, HTN, and diabetes mellitus were the most common comorbidities detected in several studies $(12,13)$. According to the results of this study, mortality hospitalization and ICU admission were not significantly influenced by remdesivir, which were in line with the results from the study by Mahajan et al where remdesivir therapy was discovered to have caused no improvement in clinical outcomes in moderate to severe COVID-19 patients (14). In another study, a group of patients received remdesivir plus corticosteroids while another group only received remdesivir. In contrast, the results of this study showed that remdesivir was associated with prompt clinical improvement in a cohort of predominantly non-White cases. remdesivir plus corticosteroid administration did not delay the death (15). This difference may have been due to the differences in genetic properties of the study population, which could have also affected the results of the study. However, there were some studies showing that the effects of remdesivir in reducing mortality and clinical symptoms of patients were unreliable (13). The trend of clinical symptoms, including gastrointestinal and other symptoms, was not significantly different between the standard and remdesivir groups; however, the prevalence of the symptoms was decreased numerically and constantly in the standard group, while the symptoms in the remdesivir group were initially decreased but later increased again. This later increase in the rate of symptoms may have been due to the side effects of remdesivir. 


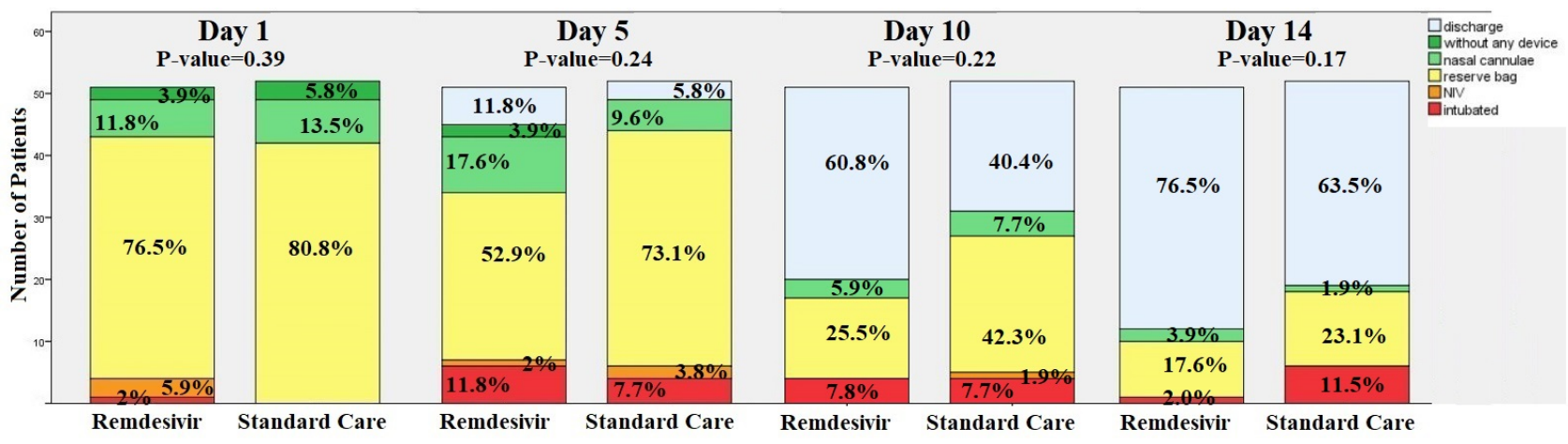

Figure 1. Respiratory status in standard and remdesivir groups.
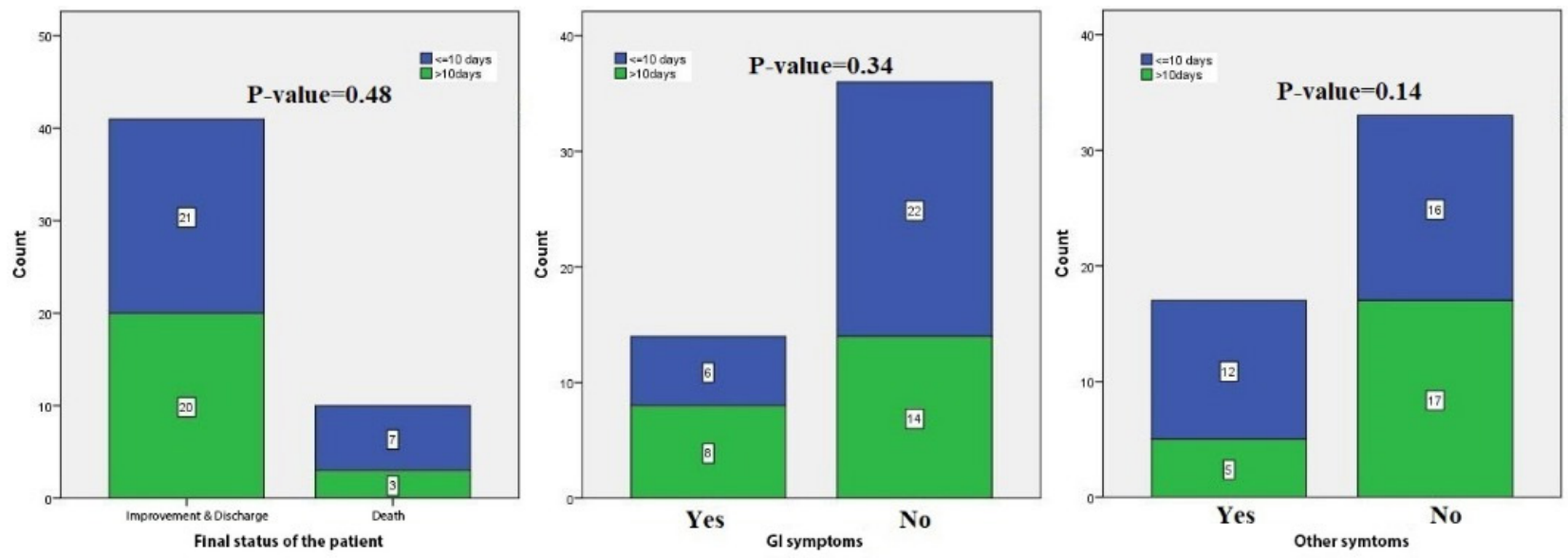

Figure 2. Mortality/discharge and gastrointestinal/other symptoms in terms of symptom onset to remdesivir treatment interval.
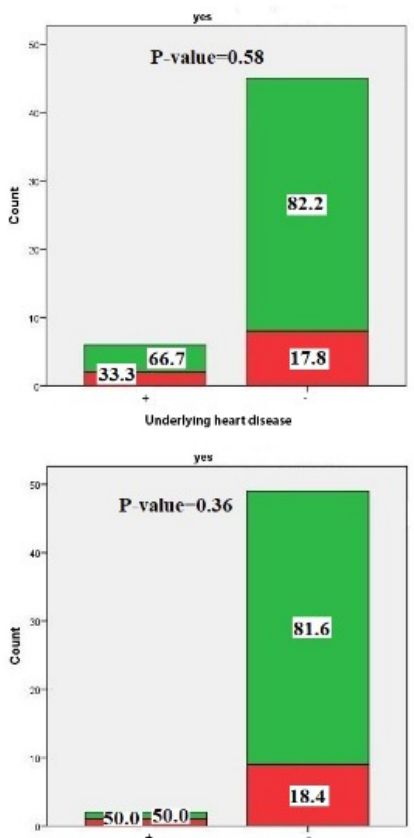

Undertying Respiratory disease
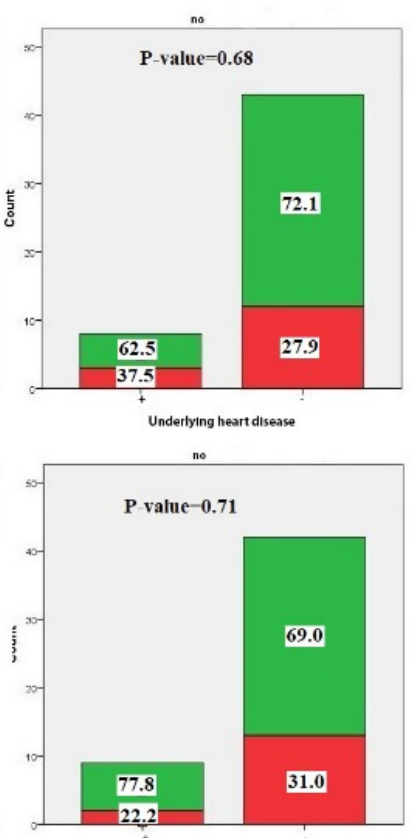

Undertying Respratory alsease

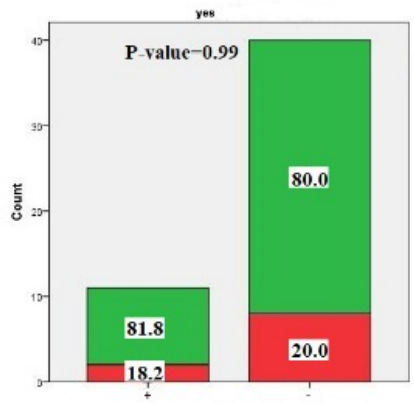

DM

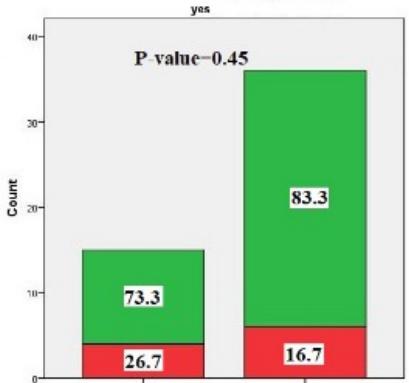

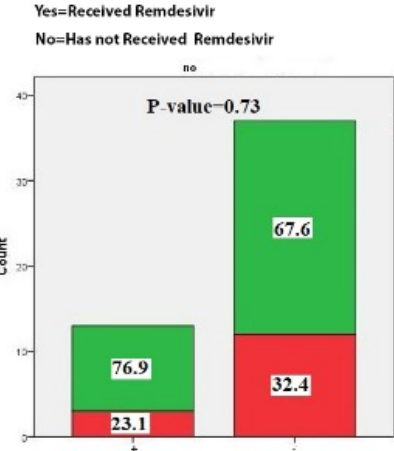

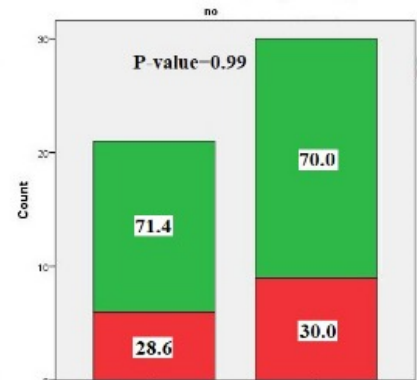

HTN

Figure 3. Mortality and discharge in term of underlying disease.

Remdesivir produced neither a significant improvement nor a decrease in lymphocyte count in the remdesivir group comparing to the standard group $(P=0.04)$. Compared to the count observed on the $1^{\text {st }}$ day, platelet count in both groups initially increased but later decreased; and these changes were only significant in the standard group. Different factors were likely responsible for the fluctuation in platelet count, specially the one concerning the application of various given drugs.

In this study, it was detected that remdesivir neither 
increased liver enzymes nor contributed to abnormal coagulation factors; according to the findings from Grein and colleagues' study, however, patients receiving remdesivir in the invasive oxygen supplementary group had higher levels of ALT and creatinine (11).

There was no significant difference between groups in terms of the patients' respiratory status. Following Tomashek and colleagues' study, however, the remdesivir group was treated with supportive oxygen for a shorter period and the incidence of supportive oxygen use in nonoxygenated cases was less in the remdesivir group (6).

The symptom onset to remdesivir therapy interval in patients in the remdesivir group was not shown to be different in the rate of recovery and mortality and gastrointestinal symptoms and myalgia and headache. Wang and colleagues' study also found no difference between the onset of symptoms and the start of treatment in terms of mortality. Goldman and colleagues however, found that patients who had symptoms less than 10 days prior to receiving remdesivir had significantly higher discharge rates compared to those who had symptoms more than 10 days between the onset of symptoms and the treatment. Tomashek and colleagues' study also demonstrated that early administration of remdesivir had more benefits. Grein and colleagues' study found no significant relationship between the mean duration of symptoms and symptom onset to the start of remdesivir treatment interval $(6,10,11)$.

No significant difference was observed regarding mortality rate affected by underlying diseases like cardiovascular disease, diabetes mellitus, HTN, and chronic respiratory disease since the most common comorbidities in both groups did not increase mortality rate.

Olender and colleagues' study identified HTN as a risk factor and the recovery rate in the non-HTN group was found to be significantly higher (9). Similar to the Goldman and colleagues' study, no significant difference was detected between our study groups regarding recovery from 5-day to 10-day courses of remdesivir treatment, whereas the recovery rate on day 14 for Olender and colleagues' study participants receiving remdesivir was two to three times higher than that for the standard group members $(9,10)$. Tomashek and colleagues' study also revealed that the remdesivir group had a shorter recovery time than the placebo group (6).

\section{Conclusion}

In this study, patients with severe COVID-19 were examined and no significant difference was found between the group receiving remdesivir and the standard care group in terms of mortality hospitalization and ICU admission. Changes in national protocols and problems regarding the lack of evidence about the confounding effects of personal variables were the limitations of the present study.

\section{Acknowledgments}

This article was derived from a research project approved by the Research and Technology Deputy of the Shahrekord University of Medical Sciences (Approval no. 5618). Hereby, the researchers would like to thank the patients who participated in this study.

Authors' Contributions

Conception and design, acquisition of data, analysis and interpretation of data were performed by AR, ZR and ZH. Drafting of the manuscript was done by MS. Critical revision of the manuscript for important intellectual content and statistical analysis were conducted by HR. The final draft was approved by AR, ZR and MS. All authors read and approved the manuscript.

\section{Conflict of Interests}

The authors declare that they have no conflict of interests.

\section{Ethical Approval}

This study protocol was approved by Shahrekord University of Medical Sciences (IR.SKUMS.REC.1399.230).

References

1. Ahmed SF, Quadeer AA, McKay MR. Preliminary identification of potential vaccine targets for the COVID-19 coronavirus (SARS-CoV-2) based on SARS-CoV immunological studies. Viruses. 2020;12(3):254. doi: 10.3390/v12030254.

2. Hassan SA, Sheikh FN, Jamal S, Ezeh JK, Akhtar A. Coronavirus (COVID-19): a review of clinical features, diagnosis, and treatment. Cureus. 2020;12(3):e7355. doi: 10.7759/ cureus.7355.

3. Wang Y, Wang Y, Chen Y, Qin Q. Unique epidemiological and clinical features of the emerging 2019 novel coronavirus pneumonia (COVID-19) implicate special control measures. J Med Virol. 2020;92(6):568-76. doi: 10.1002/jmv.25748.

4. Yan Y, Yang Y, Wang F, Ren H, Zhang S, Shi X, et al. Clinical characteristics and outcomes of patients with severe COVID-19 with diabetes. BMJ Open Diabetes Res Care. 2020;8(1). doi: 10.1136/bmjdrc-2020-001343.

5. Misra DP, Agarwal V, Gasparyan AY, Zimba O. Rheumatologists' perspective on coronavirus disease 19 (COVID-19) and potential therapeutic targets. Clin Rheumatol. 2020;39(7):2055-62. doi: 10.1007/s10067-020-05073-9.

6. Tomashek K, Dodd L. Remdesivir for the treatment of covid-19-final report. N Engl J Med. 2020;383:1813-26..

7. Sanders JM, Monogue ML, Jodlowski TZ, Cutrell JB. Pharmacologic treatments for coronavirus disease 2019 (COVID-19): a review. JAMA. 2020;323(18):1824-36. doi: 10.1001/jama.2020.6019.

8. The Ministry of Health and Medical Education. COVID-19 Patient Management Guide. Tehran: The Ministry of Health and Medical Education; 2020. Available from: https:// behdasht.gov.ir/\%D8\%B1\%D8\%A7\%D9\%87\%D9\%86 \%D9\%85\%D8\%A7\%D9\%87\%D8\%A7\%DB\%8C-\%D8 \%A2\%D9\%85\%D9\%88\%D8\%B2\%D8\%B4\%DB\%8C\%DA\%A9\%D8\%B1\%D9\%88\%D9\%86\%D8\%A7.

9. Olender SA, Perez KK, Go AS, Balani B, Price-Haywood EG, Shah NS, et al. Remdesivir for severe COVID-19 versus a cohort receiving standard of care. Clin Infect Dis. 2020. doi: 10.1093/cid/ciaa1041.

10. Goldman JD, Lye DCB, Hui DS, Marks KM, Bruno R, Montejano R, et al. Remdesivir for 5 or 10 days in patients with severe COVID-19. N Engl J Med. 2020;383(19):1827-37. doi: 10.1056/NEJMoa2015301.

11. Grein J, Ohmagari N, Shin D, Diaz G, Asperges E, Castagna A, et al. Compassionate use of remdesivir for patients with 
severe COVID-19. N Engl J Med. 2020;382(24):2327-36. doi: 10.1056/NEJMoa2007016.

12. Wang $Y$, Zhang $D, D u ~ G, D u ~ R$, Zhao J, Jin $Y$, et al. Remdesivir in adults with severe COVID-19: a randomised, double-blind, placebo-controlled, multicentre trial. Lancet. 2020;395(10236):1569-78. doi: 10.1016/s01406736(20)31022-9.

13. Spinner CD, Gottlieb RL, Criner GJ, Arribas López JR, Cattelan AM, Soriano Viladomiu A, et al. Effect of remdesivir vs standard care on clinical status at 11 days in patients with moderate COVID-19: a randomized clinical trial. JAMA.
2020;324(11):1048-57. doi: 10.1001/jama.2020.16349.

14. Mahajan L, Singh AP, Gifty. Clinical outcomes of using remdesivir in patients with moderate to severe COVID-19: a prospective randomised study. Indian J Anaesth. 2021;65(Suppl 1):S41-S6. doi: 10.4103/ija.IJA_149_21.

15. Garibaldi BT, Wang K, Robinson ML, Zeger SL, Bandeen-Roche K, Wang MC, et al. Comparison of time to clinical improvement with vs without remdesivir treatment in hospitalized patients with COVID-19. JAMA Netw Open. 2021;4(3):e213071. doi: 10.1001/jamanetworkopen.2021.3071. 